\title{
A New Method for Least-Squares and Minimax Group-Delay Error Design of Allpass Variable Fractional-Delay Digital Filters
}

\author{
Cheng-Han Chan, ${ }^{1}$ Soo-Chang Pei (EURASIP Member), ${ }^{2}$ and Jong-Jy Shyu ${ }^{3}$ \\ ${ }^{1}$ Department of Aviation and Communication Electronics, Air Force Institute of Technology, Kaohsiung 820, Taiwan \\ ${ }^{2}$ Department of Electrical Engineering, National Taiwan University, Taipei 106, Taiwan \\ ${ }^{3}$ Department of Electrical Engineering, National University of Kaohsiung, Kaohsiung 811, Taiwan
}

Correspondence should be addressed to Jong-Jy Shyu, jshyu@nuk.edu.tw

Received 28 February 2010; Accepted 22 December 2010

Academic Editor: Douglas O’Shaughnessy

Copyright (C) 2010 Cheng-Han Chan et al. This is an open access article distributed under the Creative Commons Attribution License, which permits unrestricted use, distribution, and reproduction in any medium, provided the original work is properly cited.

A double-loop iterative method is proposed to design allpass variable fractional-delay (VFD) digital filters basing on the minimization of root-mean-squared group-delay error. In the inner loop, an iterative quadratic optimization is proposed to replace the original nonlinear optimization for the minimization of root-mean-squared group-delay error, while an iterative weighting-updated technique is applied in the outer loop to further reduce the maximum group-delay error. Several examples will be presented to demonstrate the effectiveness and good convergence of the proposed method.

\section{Introduction}

For the past decade, the design of variable fractional-delay (VFD) digital filters became an important topic in digital signal processing due to their wide applications in signal processing and communication systems such as comb filter design, sample rate conversion, tunable modulator and acoustic system [1-5]. Since Farrow proposed an effective structure for implementing variable digital filter [6], several works concerning VFD filter design have been presented, including an excellent tutorial paper by Laakso, and so forth [7], FIR-based design [8-11], IIR-based design [12, 13] and allpass-based design [14-24] with their respective feature.

In this paper, the design of allpass VFD digital filters is investigated on the possible minimization of root-meansquared group-delay error. Among the existing literature in which allpass structure is applied, most applications concern the minimization of phase-oriented error, and only [23] focuses on the minimization of root-mean-squared groupdelay error by converting a nonlinear optimization problem to a linear least-squares (LS) optimization problem.

In this paper, an alternative method will be presented with comparable performance. Likely, the direct approximation of group-delay response is a highly nonlinear problem, so an iterative quadratic optimization will be proposed to overcome it in this paper. Then a weightingupdated technique $[11,25]$ is proposed to further reduce the maximum group-delay error of the designed system, which constitutes the outer loop of the overall process while the iteration stated above makes up the inner loop.

As to the stability, it has been shown in previous works [26-29] that there exists a necessary and sufficient condition for positive-valued group delay $\tau(\omega)$ of the designed allpass filter with order $N$ as follows:

$$
\int_{0}^{2 \pi} \tau(\omega) d \omega=2 \pi N .
$$

It is also pointed out in [26] that if the allpass filter design has a phase approximating error less than $\pi$ at $\omega=\pi$ it must be stable. In this paper, although there is no theoretical proof, it can be found that the designed allpass VFD filter is usually stable when mean delay of the desired response is equal to the order of the designed allpass filter and the range of adjustable parameter is properly assigned.

This paper is organized as follows. In Section 2, the review of conventional weighted least-squares (WLS) design (as Deng's method [21]) basing on the minimization of 
phase-oriented error and frequency-response-oriented error is given, and it will be shown that both will lead to the same solution. The formal formulation for LS group-delay error design of allpass VFD filters will be presented in Section 3, in which an iterative method is proposed to replace the original nonlinear optimization of group-delay-oriented error. Then in Section 4, a weighting-updated technique is proposed to further reduce the maximum group-delay error, and design examples will be given to demonstrate the effectiveness and good convergence of the proposed double-loop iterative method. Also, an example with a different range of the adjustable variable is given to show the significant effect on overall performance, which has also been revealed in $[14,24]$. Finally, the conclusions are given in Section 5.

\section{Review of Deng's Method of Allpass VFD Digital Filters}

For the design of an allpass VFD digital filter as in [21], the desired frequency response can be given by

$$
H_{d}(\omega, p)=e^{-j(N+p) \omega}, \quad|\omega| \leq \omega_{p},
$$

where $p$ is the parameter used to adjust fractional delay and $N$ denotes the order of the designed allpass filter. The transfer function of an allpass VFD digital filter is characterized by

$$
H(z, p)=z^{-N} \frac{A\left(z^{-1}, p\right)}{A(z, p)},
$$

where

$$
A(z, p)=1+\sum_{n=1}^{N} a_{n}(p) z^{-n}
$$

and the coefficients $a_{n}(p)$ are expressed as the polynomials of $p$

$$
a_{n}(p)=\sum_{m=1}^{M} a(n, m) p^{m}
$$

so (3) becomes

$$
H(z, p)=z^{-N} \frac{1+\sum_{n=1}^{N}\left(\sum_{m=1}^{M} a(n, m) p^{m}\right) z^{n}}{1+\sum_{n=1}^{N}\left(\sum_{m=1}^{M} a(n, m) p^{m}\right) z^{-n}}
$$

which can be implemented by the structure shown in Figure 1. Comparing with the structures in $[15,19]$ in which all elements are processed once for each input data, the proposed structure is designed such that the coefficient generator will generate an updated coefficient only on the demand of variation and the values of coefficients can be stored in memory, which can save enormous computation.

By (6), the frequency response of the designed system is

$$
\begin{aligned}
H\left(e^{j \omega}, p\right) & =e^{-j N \omega} \frac{A\left(e^{-j \omega}, p\right)}{A\left(e^{j \omega}, p\right)} \\
& =e^{-j N \omega} \frac{1+\sum_{n=1}^{N} \sum_{m=1}^{M} a(n, m) p^{m} e^{j n \omega}}{1+\sum_{n=1}^{N} \sum_{m=1}^{M} a(n, m) p^{m} e^{-j n \omega}}
\end{aligned}
$$

which is used to approximate (2) as much as possible over the region $R=\left\{(\omega, p), 0 \leq \omega \leq \omega_{p},-0.5 \leq p \leq 0.5\right\}$.
2.1. Phase-Oriented Approximation. Due to the unit magnitude gain for allpass filters, the design problem can focus on the phase approximation, that is, the phase of (7)

$$
\arg \left(H\left(e^{j \omega}, p\right)\right)=-N \omega-2 \arg \left(A\left(e^{j \omega}, p\right)\right), \quad(\omega, p) \in R,
$$

will be desirable to approximate the phase of (2)

$$
\arg \left(H_{d}(\omega, p)\right)=-N \omega-p \omega, \quad(\omega, p) \in R,
$$

so the error function can be represented by

$$
\begin{aligned}
e_{\theta}(\omega, p) & =\arg \left(H_{d}(\omega, p)\right)-\arg \left(H\left(e^{j \omega}, p\right)\right) \\
& =2 \arg \left(A\left(e^{j \omega}, p\right)\right)-p \omega .
\end{aligned}
$$

2.2. Frequency-Response-Oriented Approximation. An alternative view point of the design problem is the direct approximation of (2) by (7), that is, the error function is given by

$$
\begin{aligned}
e_{\mathrm{FR}}(\omega, p) & =H_{d}(\omega, p)-H\left(e^{j \omega}, p\right) \\
& =e^{-j(N+p) \omega}-e^{-j N \omega} e^{-j 2 \arg \left(A\left(e^{j \omega}, p\right)\right)} \\
& =e^{-j(N+p) \omega}\left(1-e^{-j\left(2 \arg \left(A\left(e^{j \omega}, p\right)\right)-p \omega\right)}\right) \\
& =e^{-j(N+p) \omega}\left(1-e^{-j e_{\theta}(\omega, p)}\right) \\
& =e^{-j(N+p) \omega}\left(1-\cos \left(e_{\theta}(\omega, p)\right)+j \sin \left(e_{\theta}(\omega, p)\right)\right) .
\end{aligned}
$$

For good approximation, $e_{\theta}(\omega, p) \approx 0,(\omega, p) \in R$, so

$$
\left|e_{\mathrm{FR}}(\omega, p)\right| \approx\left|e^{-j(N+p) \omega} j e_{\theta}(\omega, p)\right|=\left|e_{\theta}(\omega, p)\right|, \quad(\omega, p) \in R .
$$

Hence, both phase- and frequency-response-oriented approximations will lead to the same solution.

\subsection{WLS Solution of the Design Problem. By (10),}

$$
e_{\theta}(\omega, p)=-2 \tan ^{-1} \frac{\sum_{n=1}^{N} \sum_{m=1}^{M} a(n, m) p^{m} \sin (n \omega)}{1+\sum_{n=1}^{N} \sum_{m=1}^{M} a(n, m) p^{m} \cos (n \omega)}-p \omega
$$

which is desirable to approximate zero over $R$, and the problem can be converted into

$$
\begin{gathered}
-\frac{\sum_{n=1}^{N} \sum_{m=1}^{M} a(n, m) p^{m} \sin (n \omega)}{1+\sum_{n=1}^{N} \sum_{m=1}^{M} a(n, m) p^{m} \cos (n \omega)} \rightarrow \tan \left(\frac{p \omega}{2}\right) \\
=\frac{\sin (p \omega / 2)}{\cos (p \omega / 2)}, \quad(\omega, p) \in R
\end{gathered}
$$


where “ $\rightarrow$ " means “approximate." Equation (14) can be further replaced by

$$
\begin{aligned}
& \sin \left(\frac{p \omega}{2}\right) \\
& +\sum_{n=1}^{N} \sum_{m=1}^{M} a(n, m) p^{m}\left[\cos (n \omega) \sin \left(\frac{p \omega}{2}\right)+\sin (n \omega) \cos \left(\frac{p \omega}{2}\right)\right] \\
& \longrightarrow 0, \quad(\omega, p) \in R .
\end{aligned}
$$

Hence, the root-mean-squared objective error function for WLS design of an allpass VFD digital filter can be represented by

$$
\begin{aligned}
e_{c}(\mathbf{a}) & =\int_{-0.5}^{0.5} \int_{0}^{\omega_{p}} W(\omega)\left|\sin \left(\frac{p \omega}{2}\right)+\mathbf{a}^{T} \mathbf{b}(\omega, p)\right|^{2} d \omega d p \\
& =s_{b}+\mathbf{r}_{b}^{T} \mathbf{a}+\mathbf{a}^{T} \mathbf{Q}_{b} \mathbf{a},
\end{aligned}
$$

where $W(\omega)$ is a positive-valued weighting function, the superscript $T$ denotes the transpose operator,

$$
\begin{gathered}
\mathbf{a}=[a(1.1), \ldots, a(N, M)]^{T}, \\
\mathbf{b}(\omega, p)=\left[p\left(\cos (\omega) \sin \left(\frac{p \omega}{2}\right)+\sin (\omega) \cos \left(\frac{p \omega}{2}\right)\right), \ldots,\right. \\
\left.p^{M}\left(\cos (N \omega) \sin \left(\frac{p \omega}{2}\right)+\sin (N \omega) \cos \left(\frac{p \omega}{2}\right)\right)\right]^{T}, \\
s_{b}=\int_{-0.5}^{0.5} \int_{0}^{\omega_{p}} W(\omega) \sin ^{2}\left(\frac{p \omega}{2}\right) d \omega d p, \\
\mathbf{r}_{b}=2 \int_{-0.5}^{0.5} \int_{0}^{\omega_{p}} W(\omega) \sin \left(\frac{p \omega}{2}\right) \mathbf{b}(\omega, p) d \omega d p, \\
\mathbf{Q}_{b}=\int_{-0.5}^{0.5} \int_{0}^{\omega_{p}} W(\omega) \mathbf{b}(\omega, p) \mathbf{b}^{T}(\omega, p) d \omega d p,
\end{gathered}
$$

and the quadratic minimization of (16) will result in

$$
\mathbf{a}=-\frac{1}{2} \mathbf{Q}_{b}{ }^{-1} \mathbf{r}_{b}
$$

\section{LS Group-Delay Error Design of Allpass VFD Digital Filters}

In this section, a delay-oriented approximation for designing allpass VFD digital filters will be proposed. The desired group-delay response can be obtained by

$$
\tau_{d}(\omega, p)=-\frac{\partial}{\partial \omega} \arg \left(H_{d}(\omega, p)\right)=N+p
$$

and the actual delay response of the designed system is

$$
\begin{aligned}
& \tau_{H}(\omega, p) \\
& =-\frac{\partial}{\partial \omega} \arg \left(H\left(e^{j \omega}, p\right)\right)=N+2 \frac{\partial}{\partial \omega} \arg \left(A\left(e^{j \omega}, p\right)\right) \\
& =N-2 \frac{\left(1+\mathbf{a}^{T} \mathbf{c}(\omega, p)\right)\left(\mathbf{a}^{T} \mathbf{s}_{d}(\omega, p)\right)-\left(\mathbf{a}^{T} \mathbf{c}_{d}(\omega, p)\right)\left(\mathbf{a}^{T} \mathbf{s}(\omega, p)\right)}{\left(1+\mathbf{a}^{T} \mathbf{c}(\omega, p)\right)^{2}+\left(\mathbf{a}^{T} \mathbf{s}(\omega, p)\right)^{2}}
\end{aligned}
$$

where

$$
\begin{aligned}
& \mathbf{c}(\omega, p) \\
& =\left[p \cos (\omega), \ldots, p \cos (N \omega), \ldots, p^{M} \cos (\omega), \ldots, p^{M} \cos (N \omega)\right]^{T}, \\
& \mathbf{s}(\omega, p) \\
& =\left[p \sin (\omega), \ldots, p \sin (N \omega), \ldots, p^{M} \sin (\omega), \ldots, p^{M} \sin (N \omega)\right]^{T}, \\
& \mathbf{c}_{d}(\omega, p)=\frac{\partial}{\partial \omega} \mathbf{c}(\omega, p), \\
& \mathbf{s}_{d}(\omega, p)=\frac{\partial}{\partial \omega} \mathbf{s}(\omega, p) .
\end{aligned}
$$

Obviously, the objective error function for a delay-oriented approximation can be represented by

$$
\begin{aligned}
& e_{\tau}(\mathbf{a}) \\
& =\int_{-0.5}^{0.5} \int_{0}^{\omega_{p}} W(\omega)\left|\tau_{d}(\omega, p)-\tau_{H}(\omega, p)\right|^{2} d \omega d p \\
& =\int_{-0.5}^{0.5} \int_{0}^{\omega_{p}} W(\omega) \\
& \quad \times\left|p+2 \frac{\mathfrak{A}}{\left(1+\mathbf{a}^{T} \mathbf{c}(\omega, p)\right)^{2}+\left(\mathbf{a}^{T} \mathbf{s}(\omega, p)\right)^{2}}\right|^{2} d \omega d p,
\end{aligned}
$$

where $\mathfrak{A}$ denotes $\left(1+\mathbf{a}^{T} \mathbf{c}(\omega, p)\right)\left(\mathbf{a}^{T} \mathbf{s}_{d}(\omega, p)\right)-\left(\mathbf{a}^{T} \mathbf{c}_{d}(\omega\right.$, p)) $\left(\mathbf{a}^{T} \mathbf{s}(\omega, p)\right)$.

However, the direct minimization of (22) is highly nonlinear, so an iterative method is proposed to solve it in this section and the objective error function in the $k$ th iteration becomes

$$
\begin{aligned}
& e_{k}\left(\mathbf{a}_{k}\right) \\
& =e_{\tau, k}\left(\mathbf{a}_{k}\right)+\alpha e_{c, k}\left(\mathbf{a}_{k}\right) \\
& =\int_{-0.5}^{0.5} \int_{0}^{\omega_{p}} W(\omega)\left(A_{k-1}^{2}(\omega, p) p+2 A_{R, k-1}(\omega, p) \mathbf{a}_{k}^{T} \mathbf{s}_{d}(\omega, p)\right. \\
& \left.\quad-2 A_{I, k-1}(\omega, p) \mathbf{a}_{k}^{T} \mathbf{c}_{d}(\omega, p)\right)^{2} d \omega d p \\
& \quad+\alpha\left(s_{b}+\mathbf{r}_{b}^{T} \mathbf{a}_{k}+\mathbf{a}_{k}^{T} \mathbf{Q}_{b} \mathbf{a}_{k}\right),
\end{aligned}
$$


TABle 1: Comparison of evaluated errors in (29).

\begin{tabular}{|c|c|c|c|c|c|}
\hline Method & $\varepsilon_{\tau 2}(\%)$ & $\varepsilon_{\tau}$ & $\varepsilon_{\theta 2}(\%)$ & $\varepsilon_{\theta}$ & Design time (seconds) \\
\hline Deng's method in Section 2, $W(\omega)=1, p \in[-0.5,0.5]$ & 0.242 & 0.03145 & 0.001205 & 0.0001788 & 0.38 \\
\hline $\begin{array}{l}\text { Lee, Caccetta, and Rehbock's method [23], LS design, } \\
p \in[-0.5,0.5]\end{array}$ & 0.0992 & 0.005276 & 0.002199 & 0.0000718 & 3.19 \\
\hline Proposed LS design, $p \in[-0.5,0.5]$ & 0.1474 & 0.004137 & 0.002312 & 0.0000707 & 28.36 \\
\hline Proposed LS design, $p \in[-0.65,0.35]$ & 0.04464 & 0.001927 & 0.000724 & 0.0000543 & 28.13 \\
\hline $\begin{array}{l}\text { Lee, Caccetta and Rehbock's method [23], WLS design, } \\
p \in[-0.5,0.5]\end{array}$ & 0.155 & 0.002836 & 0.00307 & 0.0000838 & 58.63 \\
\hline Proposed minimax design, $p \in[-0.5,0.5]$ & 0.1964 & 0.002966 & 0.003235 & 0.0000834 & 148.76 \\
\hline Proposed minimax design, $p \in[-0.65,0.35]$ & 0.0664 & 0.001189 & 0.001141 & 0.0000365 & 196.56 \\
\hline
\end{tabular}

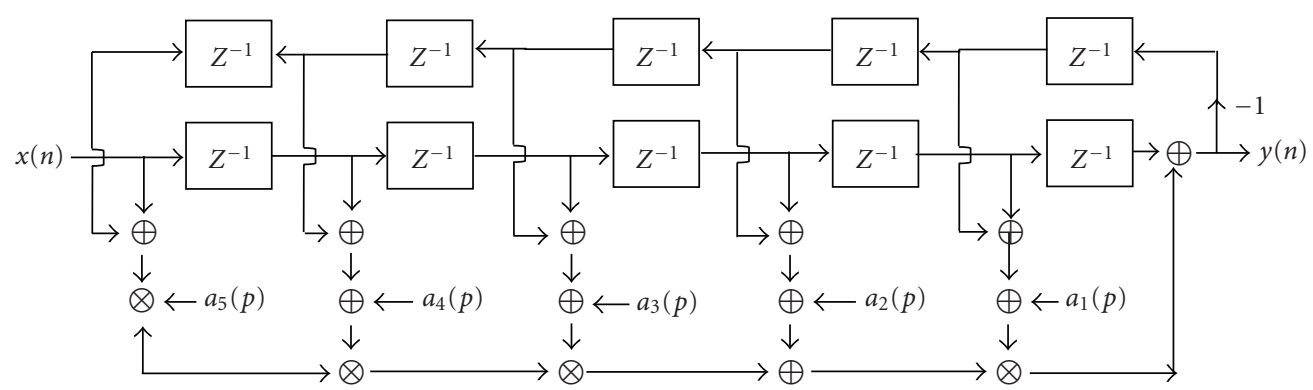

(a)

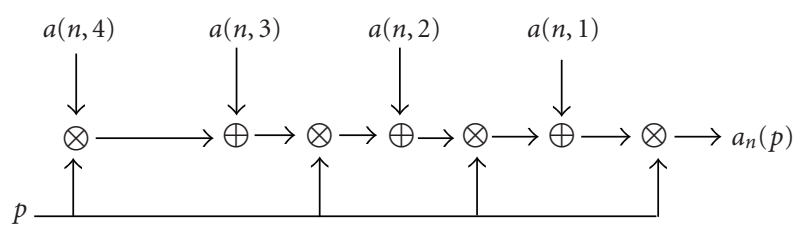

(b)

Figure 1: (a) The proposed structure of an allpass VFD digital filter $(N=5, M=4)$. (b) Coefficient generator $(1 \leq n \leq 5)$.

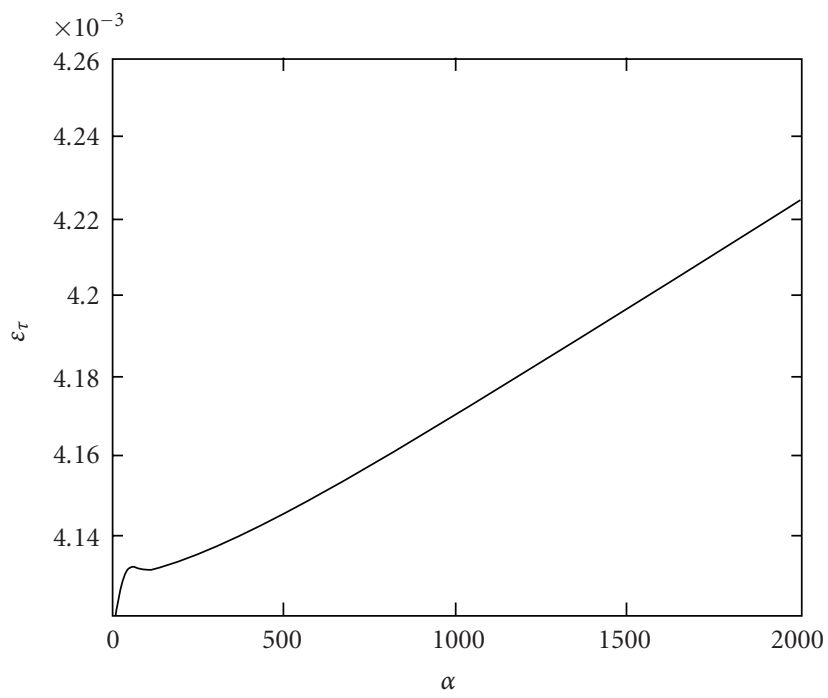

(a)

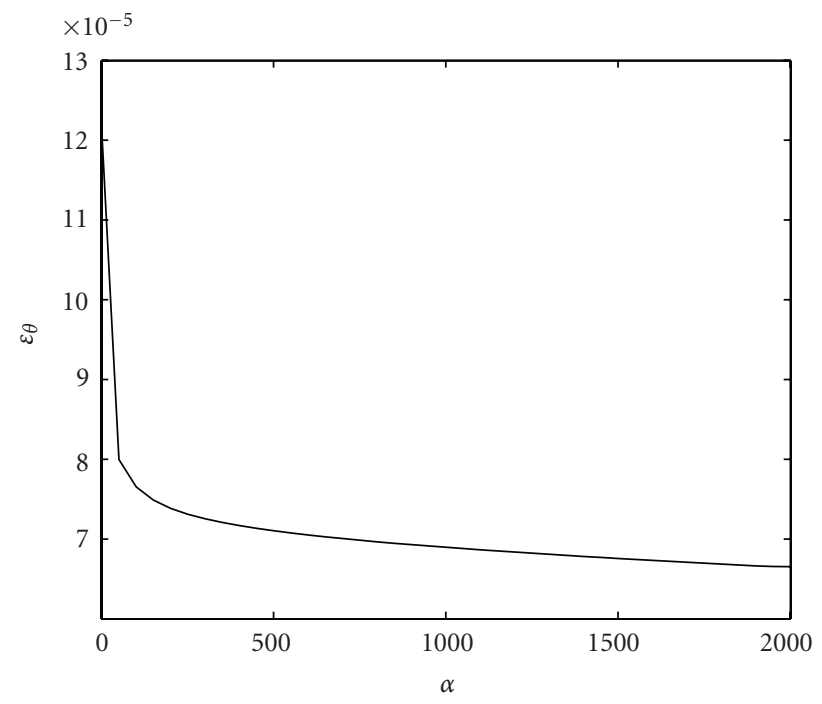

(b)

Figure 2: Curves of (a) $\varepsilon_{\tau}$ and (b) $\varepsilon_{\theta}$ when $\alpha$ varies from 1 to 2000. 
where the vector denoted by the subscript " $k$ " represents coefficient vector to be determined in the $k$ th iteration, $e_{c, k}\left(\mathbf{a}_{k}\right)$ has been likely defined in (16), $\alpha$ is a relative weighting constant, and the functions denoted by the subscript " $k-1$ " are defined by

$$
\begin{gathered}
A_{R, k-1}(\omega, p)=1+\mathbf{a}_{k-1}^{T} \mathbf{c}(\omega, p), \\
A_{I, k-1}(\omega, p)=\mathbf{a}_{k-1}^{T} \mathbf{s}(\omega, p), \\
A_{k-1}(\omega, p)=\left(A_{R, k-1}^{2}(\omega, p)+A_{I, k-1}^{2}(\omega, p)\right)^{1 / 2} .
\end{gathered}
$$

It is noted that $e_{c, k}\left(\mathbf{a}_{k}\right)$ is included in (23) and $\alpha$ must be chosen large enough to avoid the phase response of the designed system deviating from the desired one too much. Moreover, the denominator in (22) is ignored for the iterative method in (23), which will yield satisfactory results. Equation (23) can be further represented in a quadratic form as

$$
\begin{aligned}
e_{k}\left(\mathbf{a}_{k}\right)= & s_{\tau}+\mathbf{a}_{k}^{T} \mathbf{Q}_{s} \mathbf{a}_{k}+\mathbf{a}_{k}^{T} \mathbf{Q}_{c} \mathbf{a}_{k}+\mathbf{r}_{s}^{T} \mathbf{a}_{k}+\mathbf{r}_{c}^{T} \mathbf{a}_{k} \\
& +\mathbf{a}_{k}^{T} \mathbf{Q}_{c s} \mathbf{a}_{k}+\alpha\left(s_{b}+\mathbf{r}_{b}^{T} \mathbf{a}_{k}+\mathbf{a}_{k}^{T} \mathbf{Q}_{b} \mathbf{a}_{k}\right)
\end{aligned}
$$

where

$$
\begin{aligned}
& \boldsymbol{s}_{\tau}=\int_{-0.5}^{0.5} \int_{0}^{\omega_{p}} W(\omega) A_{k-1}^{4}(\omega, p) p^{2} d \omega d p \\
& \mathbf{Q}_{s}=4 \int_{-0.5}^{0.5} \int_{0}^{\omega_{p}} W(\omega) A_{R, k-1}^{2}(\omega, p) \mathbf{s}_{d}(\omega, p) \mathbf{s}_{d}^{T}(\omega, p) d \omega d p \\
& \mathbf{Q}_{c}=4 \int_{-0.5}^{0.5} \int_{0}^{\omega_{p}} W(\omega) A_{I, k-1}^{2}(\omega, p) \mathbf{c}_{d}(\omega, p) \mathbf{c}_{d}^{T}(\omega, p) d \omega d p \\
& \mathbf{r}_{s}=4 \int_{-0.5}^{0.5} \int_{0}^{\omega_{p}} W(\omega) A_{R, k-1}(\omega, p) A_{k-1}^{2}(\omega, p) p \mathbf{s}_{d}(\omega, p) d \omega d p, \\
& \mathbf{r}_{c}=-4 \int_{-0.5}^{0.5} \int_{0}^{\omega_{p}} W(\omega) A_{I, k-1}(\omega, p) \\
& \mathbf{Q}_{c s}=-4 \int_{-0.5}^{0.5} \int_{0}^{\omega_{p}} W(\omega) A_{R, k-1}(\omega, p) \\
& \quad-4 \int_{-0.5}^{2} \int_{0}^{\omega_{p}} W(\omega) A_{R, k-1}(\omega, p) p \mathbf{c}_{d}(\omega, p) d \omega d p, \\
& \times A_{I, k-1}(\omega, p) \mathbf{s}_{d}(\omega, p) \mathbf{c}_{d}^{T}(\omega, p) d \omega d p .
\end{aligned}
$$

Notice that $\mathbf{Q}_{c s}$ is so arranged that it is symmetric and positive-definite. Differentiating (25) with respect to $\mathbf{a}_{k}$ and setting the result to zero, the solution for minimizing (25) in the $k$ th iteration can be obtained as

$$
\mathbf{a}_{k}=-\frac{1}{2}\left(\mathbf{Q}_{s}+\mathbf{Q}_{c}+\mathbf{Q}_{c s}+\alpha \mathbf{Q}_{b}\right)^{-1}\left(\mathbf{r}_{s}+\mathbf{r}_{c}+\alpha \mathbf{r}_{b}\right)
$$

To terminate the iterative process, the relative norm is defined by

$$
\beta=\frac{\left\|\mathbf{a}_{k}-\mathbf{a}_{k-1}\right\|}{\left\|\mathbf{a}_{k}\right\|} .
$$

When $\beta$ is small enough, for example, smaller than $\varepsilon_{\text {inn }}$, where $\varepsilon_{\text {inn }}$ is a preassigned very small positive constant, the iterative process can stop. In this paper, $\varepsilon_{\text {inn }}=0.001$ is used. As to the initial coefficient vector $\mathbf{a}_{0}$, we can adopt the solution in (18) by setting $W(\omega)=1$. The details of iterative procedures will be described in the next section.

To evaluate the accuracy of the designed system, the normalized root-mean-squared group-delay error, the maximum group-delay error, the normalized root-mean-squared phase error, and the maximum phase error are defined by

$$
\begin{aligned}
\varepsilon_{\tau 2}= & {\left[\frac{\int_{-0.5}^{0.5} \int_{0}^{\omega_{p}}\left|\tau_{d}(\omega, p)-\tau_{H}(\omega, p)\right|^{2} d \omega d p}{\int_{-0.5}^{0.5} \int_{0}^{\omega_{p}} p^{2} d \omega d p}\right]^{1 / 2} \times 100 \%, } \\
\varepsilon_{\tau}= & \max \left\{\left|\tau_{d}(\omega, p)-\tau_{H}(\omega, p)\right|,(\omega, p) \in R\right\}, \\
\varepsilon_{\theta 2}= & {\left[\frac{\int_{-0.5}^{0.5} \int_{0}^{\omega_{p}}\left|\arg \left(H_{d}(\omega, p)\right)-\arg \left(H\left(e^{j \omega}, p\right)\right)\right|^{2} d \omega d p}{\int_{-0.5}^{0.5} \int_{0}^{\omega_{p}}(\omega p)^{2} d \omega d p}\right]^{1 / 2} } \\
& \times 100 \%, \\
\varepsilon_{\theta}= & \max \left\{\left|\arg \left(H_{d}(\omega, p)\right)-\arg \left(H\left(e^{j \omega}, p\right)\right)\right|,(\omega, p) \in R\right\},
\end{aligned}
$$

respectively. To compute (29), the frequency $\omega$ and the variable $p$ are uniformly sampled at step sizes $\omega_{p} / 200$ and $1 / 300$, respectively.

Example 1. This example deals with the proposed LS design of an $N=35, M=5, \omega_{p}=0.9 \pi$ allpass VFD filter. To properly choose $\alpha$ in (23), Figures 2(a) and 2(b) present the curves of $\varepsilon_{\tau}$ and $\varepsilon_{\theta}$, respectively, when $\alpha$ varies from 1 to 2000. In this paper, $\alpha=1000$ is used, and the design took three iterations. Figure 3(a) presents the obtained groupdelay responses while the absolute errors of group-delay and phase are shown in Figures 3(b) and 3(c), respectively, accompanying those of the Deng's method in Section 2. The related errors in (29) are tabulated in Table 1. It can be observed that both $\varepsilon_{\tau}$ and $\varepsilon_{\theta}$ of the proposed method are smaller than those of the existing method [23], but the performances of $\varepsilon_{\tau 2}$ and $\varepsilon_{\theta 2}$ for the proposed method are not as good as those in [23]. Matlab simulations show that the design took about 28.36 seconds on a notebook PC with Intel Core Duo CPU T8300.

\section{Minimax Group-Delay Error Design of Allpass VFD Digital Filters}

In this section, a weighting-updated technique is proposed to minimize the maximum group-delay error of an allpass VFD filter obtained in Section 3, which constitutes the outer loop of the overall process while the iteration in Section 3 makes 


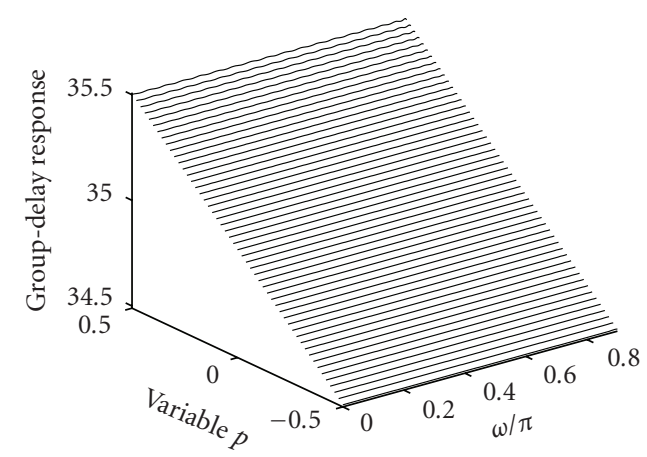

(a)
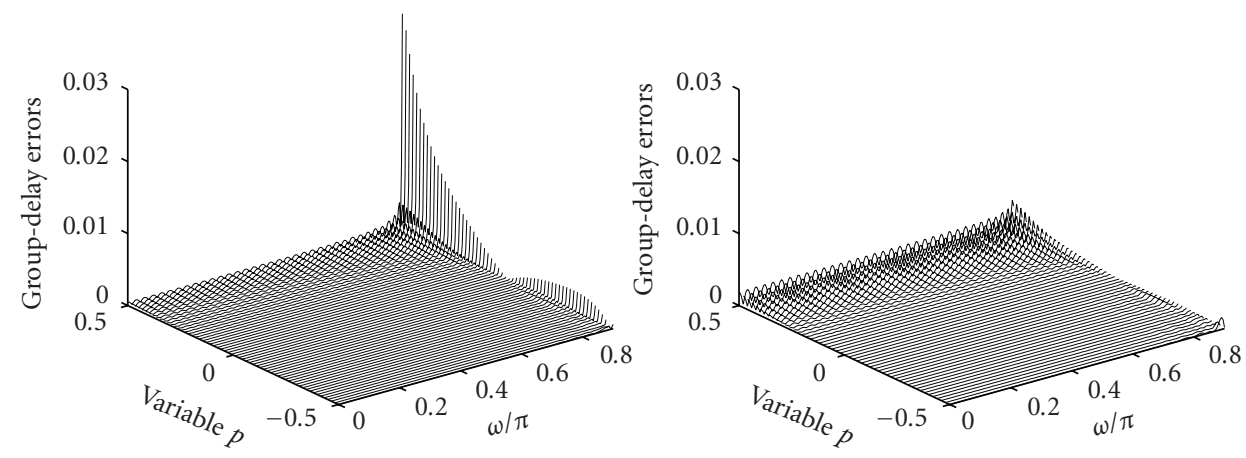

(b)
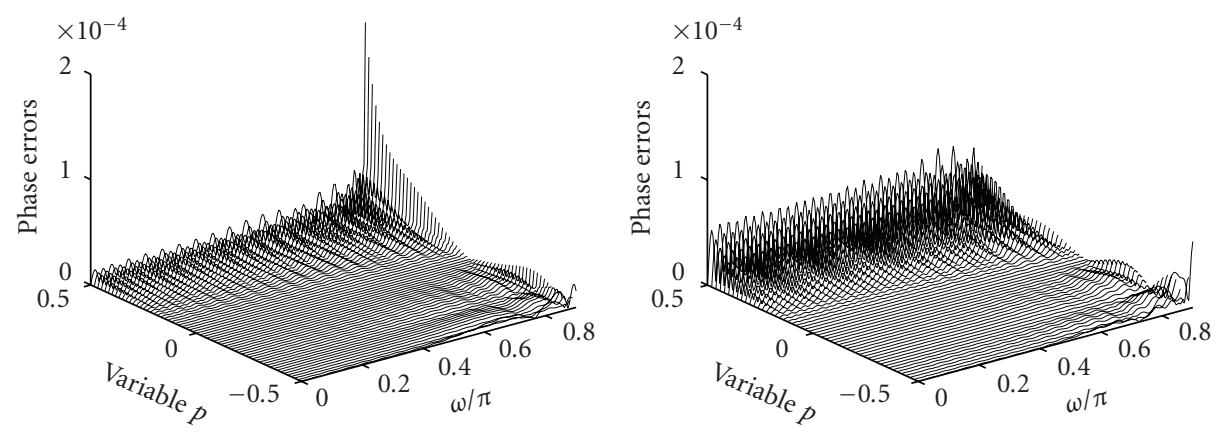

(c)

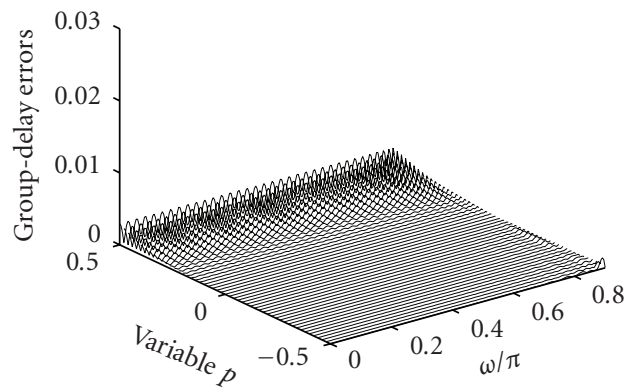

(d)

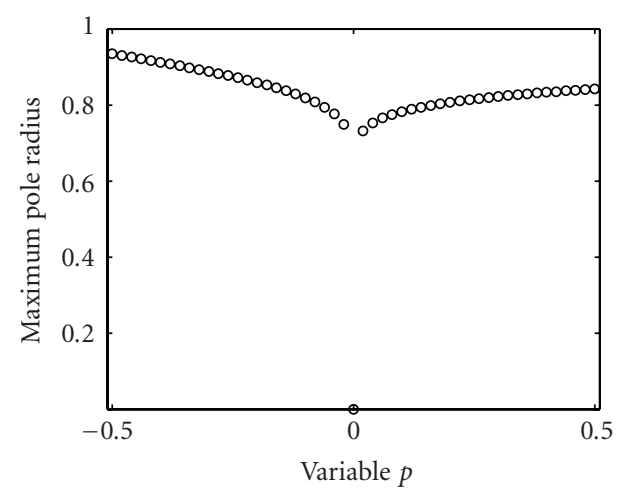

(e)

Figure 3: Design of an $N=35, M=5, \omega_{p}=0.9 \pi, p \in[-0.5,0.5]$ allpass VFD filter. (a) Group-delay responses. (b) Absolute group-delay errors (left: Deng's LS design, right: proposed LS design). (c) Absolute phase errors (left: Deng's LS design, right: proposed LS design). (d) Absolute group-delay errors of the proposed minimax design. (e) Maximum pole radius for $p \in[-0.5,0.5]$. 
TABLE 2: Filter coefficients for the proposed LS design in Example 3.

\begin{tabular}{|c|c|c|c|c|c|}
\hline \multirow[b]{2}{*}{$n$} & \multicolumn{5}{|c|}{$m$} \\
\hline & 1 & 2 & 3 & 4 & 5 \\
\hline 1 & -0.995911478379215 & 0.003037237182070 & 0.000674977600074 & 0.002203931411874 & -0.001547094931521 \\
\hline 2 & 0.491860988660958 & 0.489906840770088 & -0.004126440722118 & -0.004968604465653 & -0.000451455145871 \\
\hline 3 & -0.321238701261896 & -0.480959682281854 & -0.155527315538002 & 0.009336078160601 & 0.002875779605052 \\
\hline 4 & 0.234086131719820 & 0.429265271544100 & 0.228966726293102 & 0.025840785057596 & -0.005934120518170 \\
\hline 5 & -0.180442437563948 & -0.376964115092541 & -0.258474768641364 & -0.058885621354534 & 0.001432724569099 \\
\hline 6 & 0.143669792117880 & 0.329957770218435 & 0.265616427088872 & 0.083096234713679 & 0.005227747340939 \\
\hline 7 & -0.116657162172812 & -0.288508807455575 & -0.260564239814727 & -0.098781179155452 & -0.011492189916140 \\
\hline 8 & 0.095861039125088 & 0.251939854553747 & 0.248549917352264 & 0.107475439484688 & 0.016441996382781 \\
\hline 9 & -0.079319256800620 & -0.219538064590932 & -0.232515100469996 & -0.110719224055686 & -0.019869040384123 \\
\hline 10 & 0.065857103009291 & 0.190720077751894 & 0.214249655962206 & 0.109824100478193 & 0.021858526125015 \\
\hline 11 & -0.054726435065962 & -0.165033191450544 & -0.194914864048044 & -0.105865858472555 & -0.022611233785938 \\
\hline 12 & 0.045425714251763 & 0.142128041436134 & 0.175304729171998 & 0.099719296033186 & 0.022361719532284 \\
\hline 13 & -0.037603022298150 & -0.121728204225754 & -0.155979945905486 & -0.092096431614038 & -0.021344479562458 \\
\hline 14 & 0.031001228444685 & 0.103608784007633 & 0.137345198030118 & 0.083574882334005 & 0.019774852983403 \\
\hline 15 & -0.025425012312619 & -0.087578010616568 & -0.119690472293712 & -0.074621308011672 & -0.017844113508204 \\
\hline 16 & 0.020720915083929 & 0.073467158589459 & 0.103220353275063 & 0.065607022593667 & 0.015713946630399 \\
\hline 17 & -0.016764317587889 & -0.061120833690438 & -0.088069015325893 & -0.056822176308913 & -0.013518649356740 \\
\hline 18 & 0.013451438220377 & 0.050393631010479 & 0.074315411373739 & 0.048485405849704 & 0.011363357432515 \\
\hline 19 & -0.010693672377723 & -0.041145191376987 & -0.061990029855892 & -0.040754079903763 & -0.009328571849153 \\
\hline 20 & 0.008414281412575 & 0.033240476434287 & 0.051085766424762 & 0.033731771771784 & 0.007469846563140 \\
\hline 21 & -0.006545751741101 & -0.026547275109895 & -0.041561893474693 & -0.027476917314175 & -0.005823266602704 \\
\hline 22 & 0.005028471552094 & 0.020938283213411 & 0.033353508964552 & 0.022009510808459 & 0.004405809839568 \\
\hline 23 & -0.003809384590832 & -0.016289718620921 & -0.026374265528517 & -0.017318832133697 & -0.003221101051906 \\
\hline 24 & 0.002841516201697 & 0.012484128343807 & 0.020525052306191 & 0.013369584615560 & 0.002259936792143 \\
\hline 25 & -0.002083164017217 & -0.009409316711960 & -0.015695742339824 & -0.010108579318228 & -0.001505805273695 \\
\hline 26 & 0.001497755497971 & 0.006961232471637 & 0.011773041636065 & 0.007470070929259 & 0.000935170532813 \\
\hline 27 & -0.001053228383405 & -0.005042751228794 & -0.008641133167121 & -0.005381083473594 & -0.000522366521324 \\
\hline 28 & 0.000721982433284 & 0.003566279107085 & 0.006188479311859 & 0.003765621798479 & 0.000239360156968 \\
\hline 29 & -0.000480297868477 & -0.002452163386710 & -0.004307224739879 & -0.002548399099865 & -0.000059778977985 \\
\hline 30 & 0.000308285023293 & 0.001630854379561 & 0.002898818696283 & 0.001657749575799 & -0.000041970396936 \\
\hline 31 & -0.000189289826257 & -0.001040887457275 & -0.001872166300857 & -0.001027714173915 & 0.000087596983292 \\
\hline 32 & 0.000109818709357 & 0.000630593764138 & 0.001148101727283 & 0.000599713352933 & -0.000096409825026 \\
\hline 33 & -0.000058932273691 & -0.000355717936032 & -0.000656467669958 & -0.000323254739076 & 0.000082969883834 \\
\hline 34 & 0.000028159894437 & 0.000180788324236 & 0.000339826063819 & 0.000157105923634 & -0.000058660087578 \\
\hline 35 & -0.000010912672360 & -0.000076734250069 & -0.000151603214037 & -0.000073350022415 & 0.000026749510599 \\
\hline
\end{tabular}

up the inner loop. The overall iterative process is described in detail below.

Step 1. Given $N, M, \omega_{p}$, and $\alpha$, set $W(\omega)=1$, and find the initial coefficient vector $\mathbf{a}_{0}$ by (18).

Step 2. Set the inner iterative counter $k=0$.

Step 3. Increase the inner iterative counter $k$ by 1 , and calculate $A_{k-1}(\omega, p), A_{R, k-1}(\omega, p), A_{I, k-1}(\omega, p), \mathbf{Q}_{s}, \mathbf{Q}_{c}, \mathbf{r}_{s}, \mathbf{r}_{c}$, and $\mathbf{Q}_{c s}$.

Step 4. Find the coefficient vector $\mathbf{a}_{k}$ by (27).
Step 5. Check whether the relative norm $\beta$ is small enough by

$$
\beta<\varepsilon_{\text {inn }}
$$

If the condition is satisfied, go to the next step; otherwise go to Step 3.

Step 6. Find the variable $p$, denoted by $p_{m}$, where the maximum of group-delay error function $E(\omega, p)$, defined by

$$
E(\omega, p)=\left|\tau_{d}(\omega, p)-\tau_{H}(\omega, p)\right|, \quad(\omega, p) \in R,
$$


TABLE 3: Filter coefficients for the proposed minimax design in Example 3.

\begin{tabular}{|c|c|c|c|c|c|}
\hline \multirow[b]{2}{*}{$n$} & \multicolumn{5}{|c|}{$m$} \\
\hline & 1 & 2 & 3 & 4 & 5 \\
\hline 1 & -0.995993596236449 & 0.002951361938129 & 0.000056522430938 & 0.003227364563976 & -0.002459241277563 \\
\hline 2 & 0.492019060719535 & 0.490165494401252 & -0.002681420024658 & -0.006345896805414 & -0.000444700949259 \\
\hline 3 & -0.321471225422751 & -0.481418973949900 & -0.157782729637916 & 0.010673985821541 & 0.003558502797986 \\
\hline 4 & 0.234388948779710 & 0.429936634960839 & 0.232014003186862 & 0.024803935644968 & -0.007082100578430 \\
\hline 5 & -0.180809553898889 & -0.377848777076039 & -0.262282154813875 & -0.058330113715328 & 0.002858896290434 \\
\hline 6 & 0.144093799282764 & 0.331049886098540 & 0.270138023365234 & 0.083140840476517 & 0.003671971963968 \\
\hline 7 & -0.117129820320514 & -0.289799197810240 & -0.265745946995025 & -0.099497954673552 & -0.009920842069164 \\
\hline 8 & 0.096372854062878 & 0.253409909828576 & 0.254315329105907 & 0.108894154915106 & 0.014947469673871 \\
\hline 9 & -0.079860248869537 & -0.221163954074996 & -0.238774548835977 & -0.112836471202399 & -0.018523661245126 \\
\hline 10 & 0.066417235371802 & 0.192475127776987 & 0.220905131511245 & 0.112605063283127 & 0.020710959749977 \\
\hline 11 & -0.055295700680418 & -0.166886921064048 & -0.201858125775743 & -0.109247481340844 & -0.021690925658959 \\
\hline 12 & 0.045994562958711 & 0.144048641408887 & 0.182424468016734 & 0.103618709513285 & 0.021681689765273 \\
\hline 13 & -0.038162626126081 & -0.123683810542076 & -0.163165397984585 & -0.096416215678346 & -0.020903696245568 \\
\hline 14 & 0.031543613480551 & 0.105568113889007 & 0.144488637747372 & 0.088206732231356 & 0.019558989262805 \\
\hline 15 & -0.025943254665904 & -0.089511445430816 & -0.126690976298732 & -0.079454047897343 & -0.017831187066804 \\
\hline 16 & 0.021209234821121 & 0.075347332574665 & 0.109985232980534 & 0.070527891068195 & 0.015873812332757 \\
\hline 17 & -0.017218205011616 & -0.062923918561836 & -0.094518367293347 & -0.061725532030323 & -0.013818324456679 \\
\hline 18 & 0.013867583703075 & 0.052099069080072 & 0.080381481983181 & 0.053273169499334 & 0.011767529430927 \\
\hline 19 & -0.011069999914064 & -0.042736331042533 & -0.067618950223505 & -0.045340098661654 & -0.009802870961721 \\
\hline 20 & 0.008749913929641 & 0.034705233910870 & 0.056239697296178 & 0.038045151627557 & 0.007982131561675 \\
\hline 21 & -0.006840717660722 & -0.027876032814163 & -0.046213102316074 & -0.031459203715713 & -0.006345583922439 \\
\hline 22 & 0.005284001340328 & 0.022127902740351 & 0.037494541869108 & 0.025622074058447 & 0.004913569954169 \\
\hline 23 & -0.004027273226697 & -0.017338667620351 & -0.030005448553794 & -0.020536165254725 & -0.003696194040458 \\
\hline 24 & 0.003024238308363 & 0.013394444480610 & 0.023658566786054 & 0.016180658703661 & 0.002688753419761 \\
\hline 25 & -0.002233814412328 & -0.010187262429483 & -0.018357061617550 & -0.012518726197641 & -0.001880234103510 \\
\hline 26 & 0.001619612427739 & 0.007614467619845 & 0.013993527079620 & 0.009494927822061 & 0.001251724331496 \\
\hline 27 & -0.001149754797462 & -0.005581013645482 & -0.010458788127664 & -0.007046111678278 & -0.000781330341624 \\
\hline 28 & 0.000796663861173 & 0.004000709213585 & 0.007645263913791 & 0.005101208642714 & 0.000441439263909 \\
\hline 29 & -0.000536633718021 & -0.002795599745994 & -0.005449025857033 & -0.003589487278191 & -0.000206255609100 \\
\hline 30 & 0.000349545529838 & 0.001895830247705 & 0.003770427414620 & 0.002441776327828 & 0.000053417213621 \\
\hline 31 & -0.000218498674617 & -0.001239482351210 & -0.002517028536055 & -0.001596601179870 & 0.000032333826084 \\
\hline 32 & 0.000129607420879 & 0.000773720821702 & 0.001606542109353 & 0.000996204580929 & -0.000067169661349 \\
\hline 33 & -0.000071635910779 & -0.000454306657023 & -0.000967504900344 & -0.000587397249461 & 0.000068485823988 \\
\hline 34 & 0.000035791801597 & 0.000245674851842 & 0.000539971198304 & 0.000321069026938 & -0.000055829905847 \\
\hline 35 & -0.000015815837778 & -0.000125519073336 & -0.000300465948474 & -0.000202997633437 & 0.000015539277740 \\
\hline
\end{tabular}

occurs for the first outer iteration only. Find the absolute error ripples of $E\left(\omega, p_{m}\right)$, and denote the ith ripple with ripple interval $\left(\omega_{i-1}, \omega_{i}\right]$ by $\gamma_{i}, 1 \leq i \leq I$, where $I$ is the number of ripples in $\left[0, \omega_{p}\right]$. Then search the maximum value $\delta$ and the minimum value $\rho$ of $\gamma_{i}, 1 \leq i \leq I$.

Step 7. Check whether the error function $E\left(\omega, p_{m}\right)$ is nearly equiripple by

$$
\delta_{\rho}=\frac{\delta-\rho}{\delta}<\varepsilon_{\text {out }},
$$

where $\varepsilon_{\text {out }}$ is a preassigned very small positive constant. If the condition is satisfied, stop the process; otherwise go to the next step.
Step 8. Compute the unnormalized weighting function

$$
\widehat{W}(\omega)=W(\omega) \gamma_{i}^{2}, \quad 1 \leq i \leq I, \quad \omega_{i-1} \leq \omega \leq \omega_{i},
$$

and find its maximum value

$$
\delta_{w}=\max \left\{\widehat{W}(\omega), 0 \leq \omega \leq \omega_{p}\right\} .
$$

Then update the weighting function by

$$
W(\omega)=\frac{\widehat{W}(\omega)}{\delta_{w}}, \quad 0 \leq \omega \leq \omega_{p} .
$$

Step 9. Calculate $\mathbf{r}_{b}, \mathbf{Q}_{b}$ in (17) and replace $\mathbf{a}_{0}$ by $\mathbf{a}_{k}$. Then go to Step 2. 


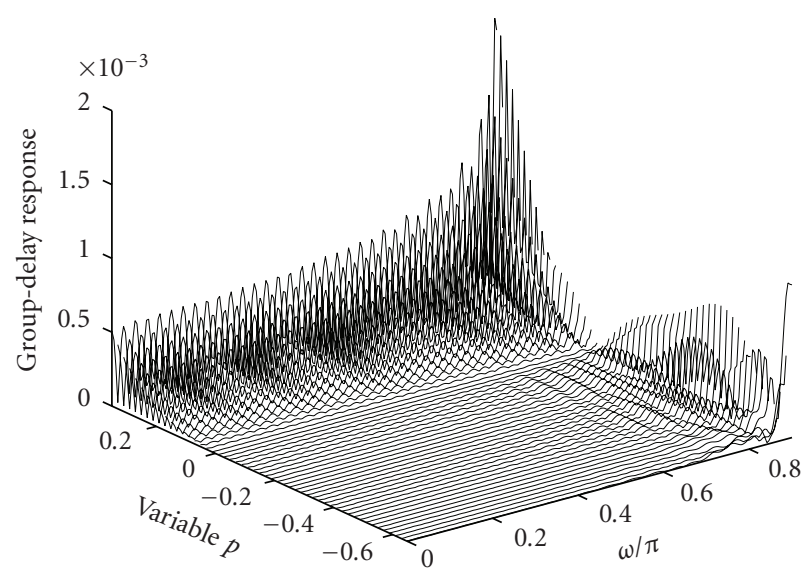

(a)

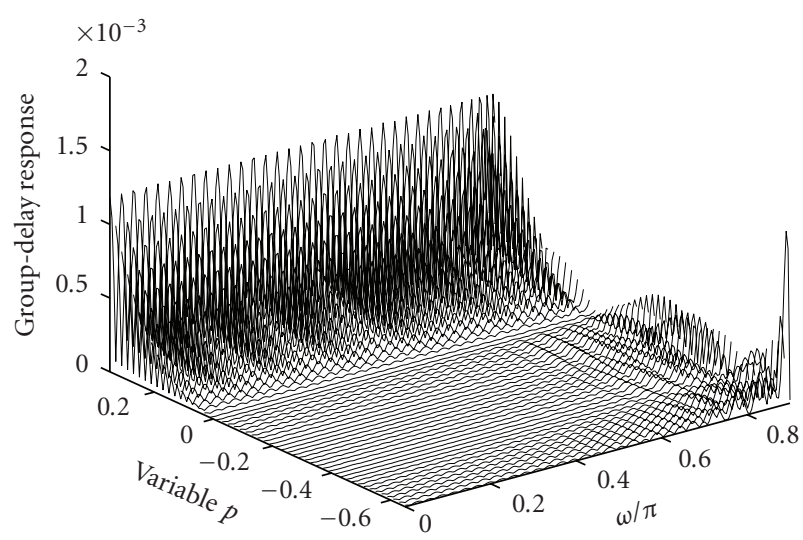

(b)

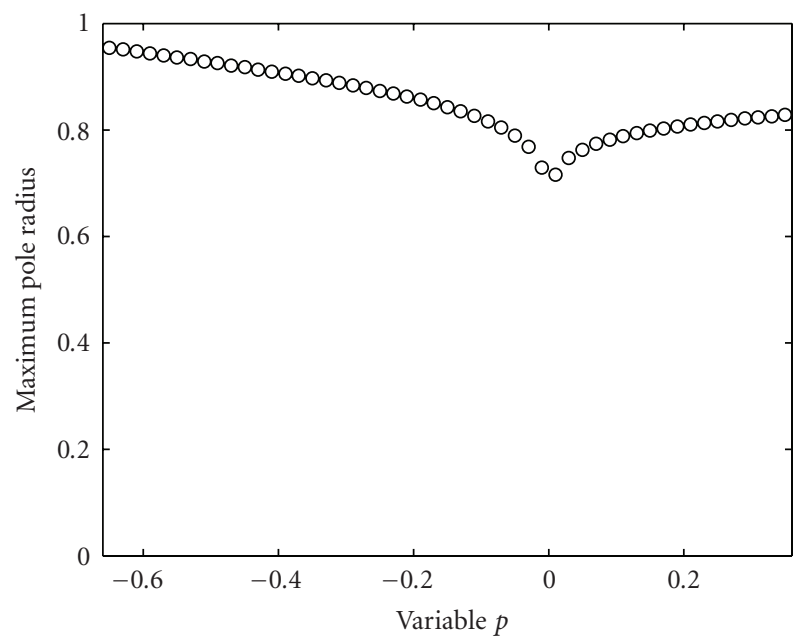

(c)

Figure 4: Design of an $N=35, M=5, \omega_{p}=0.9 \pi, p \in$ $[-0.65,0.35]$ allpass VFD filter. (a) Absolute group-delay errors of the proposed LS design. (b) Absolute group-delay errors of the proposed minimax design. (c) Maximum pole radius for $p \in$ $[-0.65,0.35]$.

Example 2. Following Example 1, the allpass VFD filter is continuously designed with minimax group-delay error. If $\varepsilon_{\text {out }}=0.01$ is used, the design took thirteen outer iterations and the respective inner iterations are three and two in the first and second outer iterations, and one in the others. Figure $3(\mathrm{~d})$ presents the final group-delay errors, and the errors computed by (29) are also listed in Table 1. To illustrate the stability of the designed filter, the maximum pole radius is shown in Figure 3(e), which shows that the designed filter is stable since the poles are all inside the unit circle for $p \in$ $[-0.5,0.5]$.

Example 3. In practice, the range of $p$ may not be limited in $[-0.5,0.5]$, and the overall performance may be even better. For example, if the allpass VFD filter is designed again with $p \in[-0.65,0.35]$ for both LS design and minimax design, the absolute errors of group-delay for LS design and minimax design are presented in Figures 4(a) and 4(b), respectively. The errors in (29) are also tabulated in Table 1, from which it can be shown that the performance of the design with $p \in$ $[-0.65,0.35]$ is much better than that with $p \in[-0.5,0.5]$. In this example, the minimax design took eighteen outer iterations, and the respective inner iterations are three and two in the first and second outer iterations, and one in the others. The final maximum pole radius is presented in Figure 4(c), which shows that the designed allpass VFD filter is stable. Also, the filter coefficients for LS and minimax designs are tabulated in Tables 2 and 3, respectively.

\section{Conclusions}

In this paper, a double-loop iterative method has been proposed to minimize the root-mean-squared group-delay error in LS and minimax senses for the design of allpass VFD digital filters. For the LS design, an iterative quadratic optimization is used in the inner loop, while a weighting-updated technique is further applied to minimize the maximum group-delay error in the outer loop. From the presented experiments, it has been shown that the performance in group delay and phase for the proposed systems can be improved drastically by appropriately specifying the range of fractional delay. For the computational complexity, although the design time of the proposed method is much more than the existing methods, an alternative method has been revealed in this paper for further research in the future.

\section{References}

[1] S. C. Pei and C. C. Tseng, "A comb filter design using fractional-sample delay," IEEE Transactions on Circuits and Systems II, vol. 45, no. 5, pp. 649-653, 1998.

[2] K. Rajamani, Y. S. Lai, and C. W. Farrow, "Efficient algorithm for sample rate conversion from CD to DAT," IEEE Signal Processing Letters, vol. 7, no. 10, pp. 288-290, 2000.

[3] R. Sobot, S. Stapleton, and M. Syrzycki, "Tunable continuoustime bandpass $\Sigma \Delta$ modulators with fractional delays," IEEE Transactions on Circuits and Systems I, vol. 53, no. 2, pp. 264273, 2006.

[4] K. J. Cho, J. S. Park, B. K. Kim, J. G. Chung, and K. K. Parhi, "Design of a sample-rate converter from CD to DAT using fractional delay allpass filter," IEEE Transactions on Circuits and Systems II, vol. 54, no. 1, pp. 19-23, 2007. 
[5] H. M. Lehtonen, V. Välimäki, and T. I. Laakso, "Canceling and selecting partials from musical tones using fractional-delay filters," Computer Music Journal, vol. 32, no. 2, pp. 43-56, 2008.

[6] C. W. Farrow, "Continuously variable digital delay element," in Proceedings of the IEEE International Symposium on Circuits and Systems, pp. 2641-2645, May 1998.

[7] T. I. Laakso, V. Välimäki, M. Karjalainen, and U. K. Laine, "Splitting the unit: delay tools for fractional delay filter design," IEEE Signal Processing Magazine, vol. 13, no. 1, pp. 30-60, 1996.

[8] H. Zhao and J. Yu, "A simple and efficient design of variable fractional delay FIR filters," IEEE Transactions on Circuits and Systems II, vol. 53, no. 2, pp. 157-160, 2006.

[9] T. B. Deng and Y. Lian, "Weighted-least-squares design of variable fractional-delay FIR filters using coefficient symmetry," IEEE Transactions on Signal Processing, vol. 54, no. 8, pp. 30233038, 2006.

[10] T. B. Deng, "Symmetric structures for odd-order maximally flat and weighted-least-squares variable fractional-delay filters," IEEE Transactions on Circuits and Systems I, vol. 54, no. 12, pp. 2718-2732, 2007.

[11] J. J. Shyu, S. C. Pei, C. H. Chan, and Y. D. Huang, "Minimax design of variable fractional-delay FIR digital filters by iterative weighted least-squares approach," IEEE Signal Processing Letters, vol. 15, pp. 693-696, 2008.

[12] H. Zhao and H. K. Kwan, "Design of 1-D stable variable fractional delay IIR filters," IEEE Transactions on Circuits and Systems II, vol. 54, no. 1, pp. 86-90, 2007.

[13] K. M. Tsui, S. C. Chan, and H. K. Kwan, "A new method for designing causal stable IIR variable fractional delay digital filters," IEEE Transactions on Circuits and Systems II, vol. 54, no. 11, pp. 999-1003, 2007.

[14] V. Välimäki, Discrete-time modeling of acoustic tubes using fractional delay filters, Doctoral thesis, Helsinki University of Technology, Espoo, Finland, 1995.

[15] M. Makundi, T. I. Laakso, and V. Välimäki, "Efficient tunable IIR and allpass filter structures," Electronics Letters, vol. 37, no. 6, pp. 344-345, 2001.

[16] M. Makundi, V. Välimäki, and T. I. Laakso, "Closed-form design of tunable fractional-delay allpass filter structures," in Proceedings of the IEEE International Symposium on Circuits and Systems, pp. 434-437, Sydney, Australia, May 2001.

[17] C. C. Tseng, "Eigenfilter approach for the design of variable fractional delay FIR and all-pass filters," IEE Proceedings: Vision, Image and Signal Processing, vol. 149, no. 5, pp. 297303, 2002.

[18] C. C. Tseng, "Design of 1-D and 2-D variable fractional delay allpass filters using weighted least-squares method," IEEE Transactions on Circuits and Systems I, vol. 49, no. 10, pp. 1413-1422, 2002.

[19] J. Yli-Kaakinen and T. Saramäki, "An algorithm for the optimization of adjustable fractional-delay all-pass filters," in Proceedings of the IEEE International Symposium on Cirquits and Systems, vol. 3, pp. 153-156, May 2004.

[20] S. C. Pei and P. H. Wang, "Closed-form design of all-pass fractional delay filters," IEEE Signal Processing Letters, vol. 11, no. 10, pp. 788-791, 2004.

[21] T. B. Deng, "Noniterative WLS design of allpass variable fractional-delay digital filters," IEEE Transactions on Circuits and Systems I, vol. 53, no. 2, pp. 358-371, 2006.

[22] H. Hacihabiboǧlu, B. Günel, and A. M. Kondoz, "Analysis of root displacement interpolation method for tunable allpass fractional-delay filters," IEEE Transactions on Signal Processing, vol. 55, no. 10, pp. 4896-4906, 2007.
[23] W. R. Lee, L. Caccetta, and V. Rehbock, "Optimal design of allpass variable fractional-delay digital filters," IEEE Transactions on Circuits and Systems I, vol. 55, no. 5, pp. 1248-1256, 2008.

[24] J. J. Shyu, S. C. Pei, and C. H. Chan, "Minimax phase error design of allpass variable fractional-delay digital filters by iterative weighted least-squares method," Signal Processing, vol. 89, no. 9, pp. 1774-1781, 2009.

[25] C. Y. Chi and Y. T. Kou, "A new self-initiated optimum WLS approximation method for the design of linear phase FIR digital filters," in Proceedings of the IEEE International Symposium on Circuits and Systems, pp. 168-171, June 1991.

[26] Z. Jing, "A new method for digital all-pass filter design," IEEE Transactions on Acoustics, Speech, and Signal Processing, vol. 35, no. 11, pp. 1557-1564, 1987.

[27] M. Lang and T. I. Laakso, "Simple and robust method for the design of allpass filters using least-squares phase error criterion," IEEE Transactions on Circuits and Systems II, vol. 41, no. 1, pp. 40-48, 1994.

[28] T. Q. Nguyen, T. I. Laakso, and R. D. Koilpillai, "Eigenfilter approach for the design of allpass filters approximating a given phase response," IEEE Transactions on Signal Processing, vol. 42, no. 9, pp. 2257-2263, 1994.

[29] K. Rajamani and Y. S. Lai, "Novel method for designing allpass digital filters," IEEE Signal Processing Letters, vol. 6, no. 8, pp. 207-209, 1999. 